\title{
Clinical Analysis of Emergency Diagnosis and Treatment of Severe Asthma with Respiratory Failure
}

\author{
Yin Haibo \\ yakeshi Forestry General Hospital of Hulun Buir, Inner Mongolia, 022150 \\ Second Affiliated Hospital of Inner Mongolia University for Nationalities, Inner Mongolia,022150
}

Keywords: Severe asthma; respiratory failure; treatment

\begin{abstract}
Objective: To analyze the successful treatment of severe asthma with respiratory failure. Methods: Retrospective analysis was performed on the hospitalization data of 30 patients with severe asthma complicated with respiratory failure. Results: The glucocorticoid methylprednisolone was administered intravenously in the early stage. The inhaled fast-acting receptor agonist and low-dose theophylline were used to relieve bronchospasm. The tracheal intubation and mechanical ventilation were given in time. All 30 patients were treated. Healed and discharged. Conclusion: Respiratory failure caused by severe asthma often threatens life and requires urgent treatment. Intravenous intubation and mechanical ventilation can improve the success rate of rescue.
\end{abstract}

Severe asthma refers to the persistence or continued deterioration of asthma symptoms after inhalation of glucocorticoids and application of long-acting receptor agonists or theophylline drugs; or asthma with fulminant episodes, shortly after asthma attacks That is, entering a critical state is often difficult to treat clinically.

\section{Data and methods}

\subsection{General information}

In this group, 30 patients were hospitalized with 18 males and 12 females, aged 28-76 years, with an average of $(481.2 \pm 12.15)$ years old. Diagnostic criteria: All cases were diagnosed in accordance with the 1993 national bronchial asthma diagnosis and severity grading standard revision program. The predisposing factors of asthma attack were 22 cases of upper respiratory tract infection and 6 cases of contact-specific allergen. The cause is unknown in 2 cases. There were 5 cases of allergic rhinitis and 8 cases of family history of asthma ${ }^{[1]}$.

\subsection{Clinical manifestations}

All patients had varying degrees of wheezing, coughing, difficulty breathing, irritability, incoherent speech, extremely severe expiratory breathing difficulties, forced sitting breathing, and sweating. A few have fever. Physical examination; 5 cases of disturbance of consciousness, 0 cases of lip purpura, can have obvious three concave signs, full thoracic, 2 cases of respiratory sound reduction, 28 cases of lung wheezing, breathing $>30$ times / min, heart rate 120-40 Times / min. Related indicators are shown in Table 1.

Table 1: Related Indicators

\begin{tabular}{|c|c|c|c|}
\hline Index & Lung Wheezing & Breathing & Heart Rate \\
\hline Quantity & 28 & 30 & $120-140$ \\
\hline
\end{tabular}

\subsection{Auxiliary inspection}

X-ray chest radiograph; 21 cases of emphysema, lung texture thickening, 9 cases of spots, punctate shadows. Arterial blood gas analysis showed hypoxemia in 30 cases, including 20 cases of 
type I respiratory failure and 5 cases of type II respiratory failure ${ }^{[2]}$.

\subsection{Treatment methods}

General treatment: All patients were given continuous low-flow oxygen inhalation, aerosolized salbutamol solution, once every half hour, 2-3 times in a row. Keep the airway unobstructed, pay attention to fluid replacement and acid-base balance, and give adequate rehydration under the condition of heart function, the amount is $2000-3000 \mathrm{ml} / 24 \mathrm{~h}$.

medical treatement. Glucocorticoid: All patients were given $80-120 \mathrm{mg}$ intravenous infusion of methylprednisolone, repeated every 6-8h. If the symptoms improved, gradually reduce the amount, and inhaled with Shuli Die powder or Pulmicort. Intravenous administration was discontinued for a week or so. 22-receptor agonist: Inhaled salbutamol solution, 2-3 times/d after symptom relief. Theophylline: aminophylline needle $0.25-0.75 \mathrm{~g} / \mathrm{d}$

Intravenous drip. Antibiotics: In the case of a history of respiratory infections, broad-spectrum antibiotics were selected based on experience before the results of drug susceptibility testing, and medications were adjusted according to efficacy and drug sensitivity test reports. For critically ill patients with type II respiratory failure, add Aichuanle solution to inhalation, every 8h. After the above treatment of arterial blood gas showed that the $\mathrm{pH}$ value was still less than 7.25 , the sputum was given $5 \%$ sodium bicarbonate $60-80 \mathrm{ml}$ intravenously once ${ }^{[3]}$.

Mechanical ventilation therapy. Five patients with respiratory distress and conscious disturbance were treated with tracheal intubation ventilator. The low-tidal volume positive end-pressure positive pressure support method was used. After 1-3 days of treatment, the patient was gradually withdrawn and extubated.

\section{Results}

Thirty patients had complete remission of asthma after comprehensive treatment, and 5 patients with disturbance of consciousness were cured and discharged.

\section{Discussion}

Airway inflammation of asthma can cause thickening of the wall, contraction of smooth muscle, and obstruction of the airway. In severe asthma, due to severe airway obstruction, the proportion of ventilated blood flow is imbalanced, causing hypoxemia and hypercapnia, pulmonary hypertension, right heart failure, leading to ventilatory failure and death. Therefore, the treatment of severe asthma should relieve asthma symptoms, improve lung function, and correct hypoxemia and hypercapnia. B2 receptor agonists can alleviate bronchial smooth muscle spasm, use salbutamol solution to inhale once every half hour, 2-3 times in a row, 5 minutes, most patients inhaled 2 times of breathing difficulties have different degrees of improvement, a few patients have improved heart rate There is a hand shake. If the patient has a history of coronary heart disease or myocardial ischemia, our experience does not allow oral beta 2 agonists to prevent severe arrhythmias and protect heart function. Glucocorticoids are considered to be the most effective anti-inflammatory drugs currently used to treat asthma. In the onset of asthma, a sufficient amount of intravenous glucocorticoid is administered to reduce the number of eosinophils, basophils and $\mathrm{T}$ cells in the circulation, regulate the release of inflammatory mediators and cytokines, reduce the secretion of bronchial mucous glands and reduce microvascular flux ${ }^{[4]}$. Permeability, in order to achieve the purpose of reducing airway inflammation and relieving paralysis. At the same time, glucocorticoids can increase the number and affinity of the $\beta 2$ receptor and increase the reactivity of the $\beta 2$ receptor. Hormone receptor levels in asthma attacks are rapidly decreasing, and the physiological effects of endogenous hormones are reduced. Therefore, the use of sufficient amounts of glucocorticoids can inhibit inflammation and stop the onset of asthma as soon as possible. Aminophylline is a better drug for the treatment of asthma.

At present, it is believed that theophylline not only has the effect of relieving airway spasm, but also inhibits the release of inflammatory mediators, and a small dose of theophylline contributes to 
the improvement of symptoms, which is consistent with our clinical practice. This group of patients was given a small dose of intravenous drip, no longer extended with traditional aminophylline intravenous bolus, all patients did not appear cardiac toxic side effects. If the patient has a history of stomach, he or she will be given a gastric mucosal protective agent. Add enough liquid to facilitate the dilution and elimination of sputum and keep the airway open. In the case of acidosis, for a solution having a $\mathrm{pH}$ of less than 7.25 after rehydration or antispasmodic treatment, a small dose of sodium bicarbonate may be administered once to maintain the $\mathrm{pH}$ at 7.25 or higher. If actively treated, asthma continues to progress to fatal ventilatory failure, tracheal intubation, mechanical ventilation should be promptly to prevent the increase of hypercapnia. Once the patient's symptoms are relieved, the tube should be removed early because the tracheal intubation itself can cause bronchospasm.

The pathophysiological changes of severe asthma are limited airflow in large and small airways, respiratory resistance is significantly increased, ventilation/perfusion ratio is imbalanced, and respiratory muscle fatigue causes diffuse atelectasis, which reduces the area of diffusion and further aggravates hypoxemia. Retention and acidosis, there is a high endogenous positive end expiratory pressure (PEEPi), mechanical ventilation is the most powerful treatment. The BiPAP ventilator assists breathing by providing bi-level positive airway pressure, which is essentially a combination of pressure-supported ventilation and continuous positive airway pressure under spontaneous breathing. Higher inspiratory pressure is used to overcome airway resistance and increase ventilation. Quantity, reduce the consumption of respiratory energy, reduce tissue oxygen consumption and carbon dioxide production. The lower expiratory pressure can resist PEEPi, to change the position of the small airway isostatic point forward, to mechanically expand the bronchus, prevent small airway collapse, effectively discharge carbon dioxide, increase lung oxygenation, improve tissue hypoxia and Clinical symptoms ${ }^{[5]}$. The application of non-invasive ventilation has developed rapidly in the past decade. Antonelli and other studies have shown that non-invasive ventilation as a first-line intervention for the treatment of respiratory failure can not only significantly reduce the use of nosocomial infections and antibiotics, but also reduce mortality. Its advantages are: 1 patient is easy to accept, greatly reducing the patient's complications (vocal cord injury, laryngeal edema, wound bleeding, ventilator-related pneumonia, etc.); 2 simple, fast, easy to master, less cost; 3 synchronization performance is good, no Affects the patient's speech and eating, maintains the airway's defense function; 4 has little effect on the circulation, and can produce beneficial hemodynamic effects and improve cardiac function on the basis of improving ventilation; 5 fewer side effects and less light, no nosocomial infection or breathing Machine pneumonia occurs.

Non-invasive ventilation is treated with mask ventilation, and there are two kinds of masks, namely nasal mask and mask. The nursing staff should choose the appropriate mask according to the individualized differences (face shape, fat and thin, respiratory condition, etc.) of the patients. The mouth is breathing, so the mask is the main one, while the patients with small and thin faces use the nasal mask. After the mask is selected, it should be fixed well, and it should be as comfortable as possible for the patient, but it should be airtight and elastic. Nursing staff actively assist patients to take appropriate postures, depending on their sitting position, sitting position or supine position, to ensure that they can open the effective airway, while ensuring the smoothness of the respiratory tract, in addition to their appropriate Adjust the cushions, etc., to actively reduce their fatigue and improve the comfort of treatment.

The condition monitoring and nursing staff should closely observe or monitor the changes in the vital signs of the patient, including respiratory rate, heart rate, body temperature, rhythm, and respiratory dynamics. If the patient's consciousness improves, the ventilation is effective, and if the patient has irritability and other emotions. Fluctuations should be promptly notified to the physician. The most critical step in non-invasive ventilation treatment is to ensure the smoothness of the respiratory tract. Therefore, the nursing staff should adjust the ventilator parameters according to the patient's condition, and at the same time ensure the patient's respiratory tract is moist. If the patient's oropharynx is dry and the sputum is not easy to discharge, then Nebulized inhalation 
treatment can be used, and the patient can be assisted in turning over the back in time to prompt them to discharge the sputum as soon as possible to ensure the smoothness of the respiratory tract. Dietary care Patients with this disease have difficulty breathing, accompanied by varying degrees of sputum and cough, which can easily accelerate their metabolism, thus losing too much water and protein. Based on this, the nursing staff should strengthen the diet care according to the patient's dietary preferences and nutritional status. Try to use high-protein, high-calorie, easy-to-digest and vitamin-rich foods, and avoid excessive intake of carbohydrates, and timely replenish water (controlled at 2500-300ml per day) to ensure effective sputum discharge.

\section{Conclusion}

In short, respiratory failure caused by severe asthma is often life-threatening and requires urgent treatment. Sufficient glucocorticoid should be used to inhibit airway inflammation. Inhaled $\beta 2$ agonist, small dose of theophylline can be used to obtain exact curative effect, timely tracheal intubation and mechanical ventilation to prevent respiratory failure and death, can improve rescue Success rate.

\section{References}

[1] Soo Jin Na,Chi Ryang Chung,Hee Jung Choi,Yang Hyun Cho,Kiick Sung,Jeong Hoon Yang,Gee Young Suh,Kyeongman Jeon. The effect of multidisciplinary extracorporeal membrane oxygenation team on clinical outcomes in patients with severe acute respiratory failure[J]. Annals of Intensive Care,2018,8(1).

[2] Jiann-Her Lin,Li-Nien Chien,Wan-Ling Tsai,Li-Ying Chen,Yung-Hsiao Chiang,Yi-Chen Hsieh. Early vertebroplasty associated with a lower risk of mortality and respiratory failure in aged patients with painful vertebral compression fractures: a population-based cohort study in Taiwan[J]. The Spine Journal,2017,17(9).

[3] Sneha Prasad,Suzanne O'Neill. Respiratory failure[J]. Surgery (Oxford),2018.

[4] SHINOZAKI Keisuke,SADAHIRO Tomohito. A Case of Myasthenia Gravis Crisis Who Required Intensive Care for Acute Respiratory Failure[J]. Journal of Tokyo Women's Medical University,2017,87(3).

[5] Ovidiu Rusalim Petris,Cristina Bologa,Victorita Sorodoc,Catalina Lionte. Repeated Bronchoscopy - Treatment of Severe Respiratory Failure in a Fire Victim[J]. The Journal of Critical Care Medicine,2017,3(4). 